\title{
Vehicle Routing Optimization for Logistics Distribution based on Artificial Fish-swarm Algorithms
}

\author{
Li Zhao \\ Shaan-xi Institute of International Trade \& Commerce Xi Xian New Area Institute of Logistics, Xi'an \\ 712046, China; \\ zhaoli0108@163.com
}

Keywords: logistics distribution; vehicle routing problem; artificial fish-swarm algorithm.

\begin{abstract}
In this paper, a method is proposed to design the optimal vehicles routing scheme for the logistics distribution. By taking the distribution cost as the objective, the vehicle routing optimization model at the case of many clients and one distributing center is established with some reasonable assumption. The artificial fish-swarm algorithm (AFSA) is a new intelligent method, which is fit to solve the NP problems with high dimensions. The principle of AFSA is presented with regard to the vehicle routing optimization model. The example shows that the optimal delivery routes can be obtained by the AFSA effectively by comparing with the particle swarm optimization.
\end{abstract}

\section{Introduction}

In recent years, more vehicles are used in logistics distribution. The vehicle routing problem becomes more complicated. It is remarkable that the unreasonable distribution scheme may affect the deliver efficiency of the logistics company, which is relative to the cost of human and material resources. The main problem is to choose the effective vehicles delivery routing by using some optimization algorithms. When performing the optimizations, the optimal routing solution can be obtained more quickly and effectively by using the artificial intelligent method, like genetic algorithms $^{[1]}$, particle swarm optimization ${ }^{[2]}$, and so on. Until now, these artificial intelligent algorithms have been applied to obtain the vehicles routing in different examples. The effectiveness is also been validated. However, it has not been used to the practice for the logistics company.

A new population-based method, Artificial Fish-Swarm algorithm (AFS), is used to solve the nonlinear optimal vehicles routing problem. The artificial fish-swarm algorithm in [3] is a new kind of swarm intelligence optimization and is presented in 2002 forward by Li Xiaolei. The research results in the domestic and foreign show that, the AFS algorithm has strong ability to escape local extremum and search for the global optimum flexibly. It is applied to solve the optimization problem in several domains, such as signal processing in [4], network combinatorial optimization in [5], and so on. Because of the little using in the vehicles delivery routing problem, the artificial fish-swarm algorithm is necessary to research its feasibility.

In this paper, we proposed a scheme by using the AFS algorithm, to fit the course characterized by excessive delivery points and complicated delivery routes. The content is organized as follows. In Section II, the logistics vehicle routing optimization model is built with minimum distribution cost; In Section III, the principle of AFS optimization is analyzed. In Section IV, an example about tobacco logistics distribution is used to demonstrate the effectiveness, and Section V is the conclusions.

\section{Model of Logistics Distribution Problem}

The Vehicle Route Problem Description. For the optimal vehicles routing problem in the logistics distribution, it is conventionally described as following. For some logistics distribution center $W_{0}$, there are $m$ vehicles to actuate the delivery assignment. The according loads are expressed as $\boldsymbol{Q}=\left[Q_{1}, Q_{2}, \cdots, Q_{m}\right]^{\mathrm{T}}$ respectively. In single dispatching period, the vehicles need to distribute the 
merchandise to $n$ clients, denoted by $W_{1}, W_{2}, \cdots, W_{n}$. The demand of these clients is expressed as $\boldsymbol{D}=\left[D_{1}, D_{2}, \cdots, D_{n}\right]^{\mathrm{T}}$, which satisfies $\sum_{i=1}^{n} D_{i} \leq \sum_{k=1}^{m} Q_{k}$. For the arbitrary dispatching process, if any vehicle $m_{r}$ needs to service for $n_{r}$ clients, then it exists $\sum_{i=1}^{n_{r}} D_{i} \leq Q_{m_{r}}$. The haul distance from the client $i$ to the client $j$ in the distribution networks is defined as $s_{i, j}$, accordingly the haul distance from distribution center to the retailers is $s_{o, i}(i, j=1,2, \cdots, n)$.

When the transport distance of the vehicles between the clients and distribution center is known, the vehicles routing can be formulated and optimized. For every client, the requirements are delivered by one appointed vehicle. For the delivery vehicles, they are limited to start from the logistics distribution center, and also end to the logistics distribution center. Commonly, the transportation cost is positive linear to the driving distance of every vehicle. The cost minimization problem is simplified to be the distance optimization problem. Therefore, the optimal vehicles routing scheme is obtained by considering those delivery constraints above with the objective of minimized transportation cost.

The Vehicle Route Model. To build mathematic model of vehicle route in logistics distribution, we make some reasonable assumptions as follow:

- There is only one available distribution center, where all the vehicles start from and return to after accomplishing the assignment.

- For arbitrary client, the delivery is achieved by a single vehicle.

- The average demand of each client is known and fixed.

- The totality of all the vehicles, which are the same type, is fixed in the distribution center.

- The time consumed by the driver rest, goods loading and goods unloading.

Before conveniently describing the relationship between the distributing vehicles and the clients, we define the following variables

$$
\Theta_{z}^{x}=\left\{\begin{array}{l}
1, \text { vehicle } z \text { distributes retailer } x . \\
0, \text { the others. }
\end{array} \Theta_{z}^{x, y}=\left\{\begin{array}{l}
1, \text { vehicle } z \text { distributes retailer } y \text { from } x . \\
0, \text { the others. }
\end{array}\right.\right.
$$

In a single dispatching period, it takes the minimum of vehicle distributing route as the optimization objective. Then the mathematic model of the vehicle route optimization in a delivery period for the logistics distribution can be described as

$$
\begin{aligned}
& \min J=\sum_{z=1}^{m} \sum_{x=0}^{n} \sum_{y=0}^{n} C_{z} \Theta_{z}^{x, y} S_{x, y} \\
& \text { s.t. } \quad \sum_{i=1}^{n_{r}} D_{i} \leq Q_{m_{r}} ; \quad m_{r}=1,2, \ldots, m \\
& \sum_{x=0}^{n} \Theta_{z}^{x, y}=\Theta_{z}^{y} ; \quad \sum_{y=0}^{n} \Theta_{z}^{x, y}=\Theta_{z}^{x} ; \quad \forall z \\
& \sum_{z=1}^{m} \Theta_{z}^{x}=1 ; \quad \Theta_{z}^{x, y}=1 \text { or } 0 ; \quad \Theta_{z}^{x}=1 \text { or } 0 ; \Theta_{z}^{y}=1 \text { or } 0 .
\end{aligned}
$$

Where the function $J$ is the objective function of the optimization, corresponding to the minimum transportation cost; the first constraint ensures that the delivery quantity is not exceeded to the load limitation for every vehicle; the second constraint is used to guarantee that the start and end point is the logistics distribution center in the single-to-single distribution; and the third constraint is used to limit the client to be dispatched by only one vehicle.

\section{Artificial Fish-Swarm Algorithm}

Algorithm Description. Artificial fish-swarm algorithm is an animal population based on the optimization strategy with the simple bottom-up design structure. Through simulating the based on the fishes' foraging, cluster and trace behavior of the fish-swarm in the nature. The artificial fish swarm algorithm has many advantages, which can be concluded. (1) the parallel calculation: the 
fishes in the swarm swim forward to the global solution in parallel with each other. (2) the simple structure: the algorithm only uses the function value of the objective as the decision data. (3) the global searching: it can escape out of the local optimal solution easily. (4) the fast calculation: the algorithm can research the best position fast even with some random factors.

The dimension search space of the fish swarm is $p$. The population of fishes in the swarm is denoted with $q$, which form the multidimensional space $\boldsymbol{X}=\left\{\boldsymbol{X}_{1}, \boldsymbol{X}_{2}, \cdots, \boldsymbol{X}_{q}\right\}$. And the according position and velocity of the $i$-th particle can be expressed as $\boldsymbol{X}_{i}=\left(X_{i 1}, X_{i 2}, \cdots, X_{i p}\right)^{\mathrm{T}}$. AFS algorithm is attempting to find an optimal location with respect to a fitness function $\boldsymbol{Y}$. The fitness function is corresponding to the food concentration of the fish position $\boldsymbol{X}_{i}$. It can be described as $\boldsymbol{Y}_{i}=f\left(\boldsymbol{X}_{i}\right)$. We define the best previous position of a fish (local best) in the swarm as $\boldsymbol{P}_{i}=\left(P_{i 1}, P_{i 2}, \cdots, P_{i p}\right)^{\mathrm{T}}$, and define the best position of a particle (global best) as $\boldsymbol{P}_{g}=\left(P_{g 1}, P_{g 2}, \cdots, P_{g p}\right)^{\mathrm{T}}$, such that $\boldsymbol{Y}_{i}=f\left(\boldsymbol{X}_{i}\right)$ is a decline function about the local best $\boldsymbol{P}_{i}$ and the global best $\boldsymbol{P}_{g}$.

Evolution Course. The evolution course of artificial fish-swarm algorithm is constituted as follows.

(1) the fishes' foraging behavior: For the artificial fish $i$, the position state is $\boldsymbol{X}_{i}$. We can choose the other artificial fish $j$ in the visual scope of the fish $i$, whose position state is $\boldsymbol{X}_{j}$. If the food concentration $\boldsymbol{Y}_{j}$ of the artificial fish $j$ is bigger than that of the artificial fish $i$, the local fish $i$ swims to the neighbor fish $j$. The position state $\boldsymbol{X}_{i}$ is updated as

$$
\boldsymbol{X}_{\text {next }}=\frac{\boldsymbol{X}_{j}-\boldsymbol{X}_{i}}{\left\|\boldsymbol{X}_{j}-\boldsymbol{X}_{i}\right\|} \cdot \text { step } \cdot r
$$

where $\boldsymbol{X}_{\text {next }}$ is the position of the next generation, $r$ is a random factor in [-1,1], step is the swimming distance. Otherwise, the neighbor fish $\boldsymbol{X}_{j}$ is chosen in the visual scope randomly. The condition about the food concentration is judged again to determine the next generation. If the number of the foraging behavior is reach to the maximum, the artificial fish $i$ will actuate the random behavior.

(2) the fishes' cluster behavior: We can choose the other artificial fishes in the visual scope of the fish $i$, and the center of these fishes is calculated denoting by $\boldsymbol{X}_{j}$. If the corresponding food concentration $\boldsymbol{Y}_{j}$ satisfies $\boldsymbol{Y}_{j} / n_{f}>\delta \boldsymbol{Y}_{i}$, the ambient of the center fish has enough food and the fishes does not congest. The variable $n_{f}$ is the number of fish in the neighborhood, $\delta$ is the congest factor. The position state $\boldsymbol{X}_{\boldsymbol{i}}$ is updated as (3). Otherwise, the fish $i$ will actuate the random behavior.

(3) the fishes' trace behavior: We can choose the other artificial fishes in the visual scope of the fish $i$. Then the fish position with maximum food concentration is calculated denoting by $\boldsymbol{X}_{j}$. If the food concentration $\boldsymbol{Y}_{j}$ satisfies $\boldsymbol{Y}_{j} / n_{f}>\delta \boldsymbol{Y}_{i}$, the ambient of the center fish has enough food and the fishes does not congest. The position state $\boldsymbol{X}_{\boldsymbol{i}}$ is updated as (3). Otherwise, the artificial fish $i$ will actuate the random behavior.

\section{Numerical Simulation and Validation}

To validate the effectiveness of the fish swarm algorithm, we choose a city with 16 fixed clients points in [6] as the example. The network constructed by the distributing route is shown in Figure 2. There are probable $6.22 \times 10^{9}$ distributing routes in the case of all the clients points finishing delivery. Therefore, it is particularly important to design an effective distributing route in a short time. The requirement of each retailer is shown in [6].

The population of the swarm is initialized as 50 . The maximum iteration is 400 . Because the VRP is a minimal value problem, the food concentration of each fish is taken the negative, and the initial value is set as -10000 . The congest factor is 0.618 , and the maximum try number is 100 . The visual scope of each fish is 6 , swimming step is 2 . 
To illuminate the effectiveness of AFS, the calculation is compared with particle swarm optimization. The local best fitness and the global best fitness will be obtained in every generation. The convergence curves of the fitness about the above two methods is shown in Figure 3.

According to Figure 2, we can obtain that the best vehicles routing generated by the AFS is much smaller than that of the PSO. It illuminates the global optimum of the combined nondeterministic polynomial problem in logistics distribution. It also can obtain that the convergence curve of the AFS is much slower than the PSO. In the beginning, the fitness of PSO is declined to the best value much faster than that of AFS. But it converged into the local optimal value at the point $f=474$. On the other hand, the global value generated by AFS is the point $f=459$. All the results mean that the convergence velocity needs to be enhanced, especially for the large scale optimization problem.

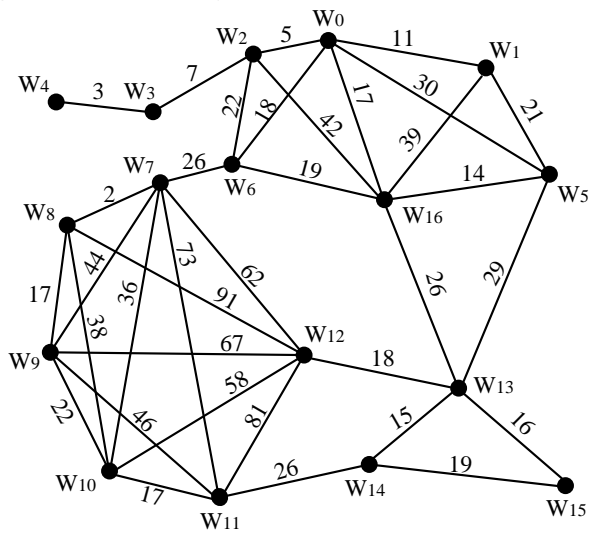

Fig.1 The clients network.

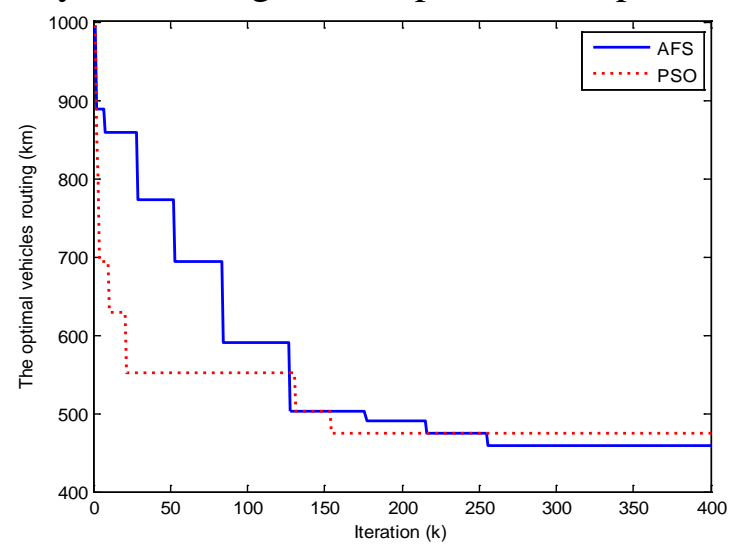

Fig. 2 The convergence curves of the fitness.

The according best vehicle routes for logistics distribution generated by AFS are shown in Table 1.

Table 1 The Best Vehicle Route

\begin{tabular}{|c|c|c|c|}
\hline Vehicles & The best distributing route & Distance & Load \\
\hline 1 & $\mathrm{~W}_{0} \rightarrow \mathrm{W}_{1} \rightarrow \mathrm{W}_{5} \rightarrow \mathrm{W}_{13} \rightarrow \mathrm{W}_{14} \rightarrow \mathrm{W}_{15}{ }^{*} \rightarrow \mathrm{W}_{12}{ }^{*} \rightarrow \mathrm{W}_{0}$ & 190 & 200 \\
\hline 2 & $\mathrm{~W}_{0} \rightarrow \mathrm{W}_{7} \rightarrow \mathrm{W}_{8} \rightarrow \mathrm{W}_{9} \rightarrow \mathrm{W}_{10} \rightarrow \mathrm{W}_{11} \rightarrow \mathrm{W}_{0}$ & 186 & 203 \\
\hline 3 & $\mathrm{~W}_{0} \rightarrow \mathrm{W}_{2} \rightarrow \mathrm{W}_{3} \rightarrow \mathrm{W}_{4} \rightarrow \mathrm{W}_{6} \rightarrow \mathrm{W}_{16}{ }^{*} \rightarrow \mathrm{W}_{0}$ & 83 & 204 \\
\hline
\end{tabular}

\section{Summary}

The vehicle routes optimization in logistics distribution is researched in this paper, by using the artificial fish-swarm algorithm. The mathematical model of the vehicle routes optimization is built by taking the vehicle cost as the objective. Focusing on the combined nondeterministic polynomial problem, the AFS algorithm is developed. Through simulating the fishes' foraging, cluster and trace behavior in the calculation, the global optimal solution is obtained effectively. Finally, an example with 16 distributing clients of a city is used to validate the effectiveness of the improved method.

\section{References}

[1] J. McCall, Genetic algorithms for modelling and optimization. Journal of Computional and Applied Mathematics, 184(2005), pp. 205-222.

[2] J. Kennedy, R.C. Eberhart, Particle swarm optimization. Proc. of IEEE International Conference on Neural Networks, 1995, pp. 1942-1948.

[3] Xiaolei Li, Zhijiang Shao, Jixin Qian, An optimizing method based on autonomous animates: fish-swarm algorithm. Systems Engineering Theory \& Practice, 22(2004), pp.32-38.

[4] M.Y. Jiang, D.F.Yuan, Wavelet threshold optimization with artificial fish swarm algorithm. Proc. of IEEE International Conference on Neural Networks and Brain, Beijing, 13(2005), pp.569 -572. 
[5] X.J. Shan, M.Y. Jiang, The routing optimization based on improved artificial fish swarm algorithm. Proc. of World Congress on ICA, Dalian, 12(2006), pp. 3658-3660.

[6] Zhao Li, Strategy for the optimization of tobacco distribution based on QPSO. Logistics technology, 222(2010), pp. 86-88. 